Service social

\title{
Plus ça change, plus c'est pareil : le changement et l'approche de thérapie brève
}

\section{Richard Kouri}

Volume 35, numéro 1-2, 1986

Recherche - Action - Évaluation

URI : https://id.erudit.org/iderudit/706304ar

DOI : https://doi.org/10.7202/706304ar

Aller au sommaire du numéro

Éditeur(s)

École de service social de l'Université Laval

ISSN

1708-1734 (numérique)

Découvrir la revue

Citer cet article

Kouri, R. (1986). Plus ça change, plus c'est pareil : le changement et l'approche de thérapie brève. Service social, 35(1-2), 246-260.

https://doi.org/10.7202/706304ar d'utilisation que vous pouvez consulter en ligne.

https://apropos.erudit.org/fr/usagers/politique-dutilisation/ 
KouRI, Richard, coordonnateur du module social C.L.S.C., Centre de services sociaux de l'Abitibi-Témiscamingue, à Amos.

\section{Plus ça change, plus c'est pareil : le changement et l'approche de thérapie brève}

\section{Richard Kouri}

Il n'est plus besoin de démontrer qu'un des objectifs fondamentaux de l'intervention d'aide est d'induire le changement. Et la pratique clinique témoigne quotidiennement de la réalité du changement thérapeutique. Pour assurer et faciliter celui-ci, il n'est plus besoin non plus de démontrer les efforts incessants et renouvelés que mettent les intervenants à développer et raffiner leurs méthodes d'intervention. Enfin, nos connaissances évoluent rapidement et l'arsenal des moyens d'intervention ne cesse d'augmenter et de gagner en efficacité.

L'intervenant social se heurte toutefois régulièrement à des questions sur la valeur réelle, la signification, la pertinence, la qualité, la durabilité, etc., des changements opérés chez le client. En d'autres mots, les changements opérés en sont-ils vraiment? C'est d'ailleurs avec un sourire aux lèvres, des hochements de tête affirmatifs et un haussement d'épaules désabusé que les personnes concernées répondent à cette question et mettent fin à toute discussion sur le sujet en disant : "Plus ça change, plus c'est pareil! ». Ce vieil adage relève de la sagesse populaire, qui renferme souvent de profonds éléments de vérité; d'ailleurs, la vie courante est pleine d'illustrations qui en confirment la valeur toujours actuelle.

Comment l'intervenant social doit-il ou peut-il réagir à cette contradiction apparente entre son rôle et sa réalité d'agent de changement, et face au scepticisme cynique dont la sagesse populaire fait preuve face au changement? Celle-ci condamne-t-elle donc l'intervenant social à la fabrication de mirages dont la mise en marché serait assurée par Don Quichotte? Ou ne contient-elle pas, comme à 
son habitude d'ailleurs, des subtilités dont la finesse mérite plutôt d'être percée? Cet article postule que cet adage recèle des vérités qu'il faut clarifier si l'intervenant social souhaite en éviter les pièges.

Le dicton précité souligne avec force, qu'en dépit de leur apparence d'opposition, deux réalités sont indissociables : la stabilité et le changement. Cet article tentera de dégager les mécanismes qui associent et distinguent à la fois ces deux réalités, à la lumière de la théorie du changement sur laquelle repose l'approche de thérapie brève développée à Palo Alto. Plusieurs éléments justifient ce choix. D'abord et avant tout, ses représentants les plus connus font explicitement reposer leurs stratégies d'intervention sur des fondements théoriques qui s'adressent directement au défi que soulève l'adage énoncé plus haut (Watzlawick, 1974: 1). De plus, il s'agit d'une démarche centrée sur la résolution de problèmes et qui vise une atteinte rapide de résultats. Sur un plan pratique, ces promesses de rendement (la résolution de problèmes et l'obtention de résultats) et d'efficacité (la brièveté des interventions) méritent l'intérêt des professionnels de la relation d'aide, particulièrement ceux du secteur parapublic. Nos sociétés contemporaines sont marquées par l'envahissement de l'appareil gouvernemental dans la vie privée, y compris la prestation des services socio-sanitaires; il peut donc être opportun d'en réduire la place autant que faire se peut. Les interventions brèves peuvent-elles offrir, à l'intervenant social, l'occasion de se retirer plus rapidement de la vie privée des clients après y avoir effectué les changements nécessaires?

Enfin, et contrairement aux autres approches d'intervention en travail social, celle développée à Palo Alto doit son existence à l'influence déterminante qu'exerça l'anthropologue culturel Gregory Bateson auprès de quelques psychiatres ${ }^{1}$. II s'agit-là d'un inédit en travail social et qui promet d'offrir à l'intervenant social une source différente et d'inspiration plus sociologique. Cet article examinera donc la théorie du changement à l'aide de cette approche en cherchant à en dégager et à en comprendre les fondements théoriques. Après avoir esquissé rapidement la théorie du changement que propose le groupe de Palo Alto, nous nous attarderons sur les trois fondements théoriques majeurs qui appuient et expliquent le changement. Et, tout au long du présent travail, la préoccupation centrale sera de cerner en quoi est exact l'adage " plus ça change, plus c'est pareil » et aussi en quoi il ne l'est pas. La démarche sera résolument abstraite et théorique, sur la foi qu'il n'y a rien de plus pratique qu'une bonne théorie. 


\section{Théorie du changement}

Comprendre la théorie du changement proposée par le groupe de Palo Alto, c'est surtout comprendre qu'un problème n'est rien d'autre que sa solution.

\section{L'origine d'un problème ${ }^{2}$}

Dans le cours normal de leurs activités, les gens doivent affronter des difficultés qui sont des événements inusités ou inattendus (maladie, perte d'emploi, etc.). Le plus souvent, par contre, elles proviennent de la nécessité de s'adapter aux périodes de transition que présentent les étapes du cycle de vie (mariage, naissances, scolarisation, adolescence et départ des enfants, retraite, décès du conjoint, etc.).

La plupart des gens réussissent à les régler relativement bien et, pour ce faire, ont recours à des solutions qui relèvent du sens commun ou de la sagesse populaire qui relèvent, eux, de la logique et s'appuient souvent sur des mesures approuvées par la tradition ou des conventions sociales bien établies. Habituellement, la solution consiste à combattre ou à remplacer la difficulté par son contraire, comme c'est le cas par exemple avec la chaleur pour combattre le froid.

Pour qu'une "difficulté" de la vie courante évolue en un "problème" susceptible de bénéficier d'une intervention d'aide, il suffit d'abord qu'elle ne soit pas résolue correctement et, qu'ensuite, les mêmes tentatives de solution soient remises à l'essai : une tentative de solution infructueuse et inefficace au départ n'est pas correctement reconnue comme telle et les personnes impliquées insistent et s'acharnent pour régler la difficulté toujours de la même manière, comme si c'était la seule valable. C'est donc par l'application du principe "du pareil au même " que les problèmes surgissent. En d'autres mots, le problème, c'est sa solution : plus les gens tentent de changer une situation, plus elle reste pareille.

Ce qui reste à comprendre sur l'origine des problèmes, c'est comment quelqu'un puisse appliquer des solutions « qui ne fonctionnent pas " et qui peuvent même aggraver une situation déjà assez difficile! Dès le début de notre vie, nous apprenons tous des façons habituelles, acceptées et efficaces pour régler ce que nous apprenons aussi à reconnaître comme des difficultés. Lorsqu'un effort a été fourni et que "cela n'a pas marché ", ce n'est pas à dessein ou volontairement que cela se produit. Les gens vont chercher à régler une difficulté en ayant recours à une mesure qui correspond à leur vision du monde, leurs 
croyances, leur morale et leurs opinions. Ils vont essayer telle ou telle autre mesure, convaincus qu'elle est "logique», «nécessaire» ou "la seule chose à faire ".

Plus souvent qu'autrement, ces mesures "fonctionnent" et facilitent une solution. Mais lorsque ces efforts échouent, les personnes $\mathrm{y}$ voient très facilement une confirmation de la gravité du problème et vont conclure au besoin de répéter encore et encore, avec plus de vigueur et plus d'énergie, les mêmes mesures (Segal, 1982: 280). Les façons que nous avons de régler des difficultés sont largement tributaires de nos schèmes culturels et sociaux, et ont donc été "apprises" à un niveau qui échappe à la conscience courante et explicite. II devient alors très difficile de remettre en question et de modifier les normes qui influencent nos comportements. Et, dans une situation difficile, que nous ne parvenons pas à régler, nous aurons même tendance à "raidir" nos comportements et à recourir plus spontanément encore à ces mesures "consacrées".

L'exemple de l'insomnie permettra d'illustrer clairement la position décrite ci-dessus. L'insomniaque est quelqu'un qui, au départ, a commencé à éprouver des difficultés à dormir. Pour régler ce problème, il cherchera à provoquer le sommeil en agissant, par exemple, sur les conditions qui le favorisent : il surveillera donc ses habitudes alimentaires, ses activités et son rythme de vie, et si ces mesures devaient échouer, il pourra même recourir à des médicaments : la situation peut se détériorer, et, dans ce cas, la personne pourra développer une dépendance aux médicaments, en consommer plus fréquemment et augmenter leur puissance. Ce que doit affronter l'insomniaque, c'est de s'endormir, c'est-à-dire passer de l'état d'éveil au sommeil, et son erreur sera de "trop le vouloir » ou trop "vouloir le provoquer" par des actes volontaires; c'est comme s'il voulait remplacer son état d'éveil par son contraire : le sommeil. Et, si agir sur les conditions favorisant le sommeil (alimentation et rythme de vie) réussit à la grande majorité des gens, celui pour qui cela ne réussit pas et qui tente encore davantage de s'endormir par divers actes volontaires y parviendra encore moins, précisément parce que sa recherche d'une solution est de trop chercher le sommeil. Plus l'insomniaque tente de modifier son état d'éveil, plus il restera éveillé... et son problème n'est rien d'autre que son effort à le régler.

\section{L'issue}

Pour résoudre son problème, l'insomniaque devra réviser ses prémisses et sortir du cadre de référence : il lui suffira probablement de 
chercher plutôt à rester éveillé pour que le sommeil, phénomène spontané, le gagne probablement facilement. Chercher à rester éveillé pour s'endormir paraît aller à l'encontre du sens commun, illogique et paradoxal; mais cela fonctionne, et fait ainsi mentir l'adage "plus ça change, plus c'est pareil».

C'est donc en s'attaquant aux "solutions» expérimentées par le client plutôt qu'à son "problème " que le groupe de Palo Alto propose de régler les problèmes, et ce en proposant au client des comportements qui rompent les cercles vicieux dans lesquels il s'enlisait.

Au premier abord, cette théorie paraît presque trop simple et banale. À la réflexion, toutefois, il faut reconnaître que le rôle de l'intervenant sera d'amener le client à adopter des comportements qui lui apparaîtront comme absurdes, illogiques et ridicules... Et voilà qui complique tout. À ce point-ci, il serait fascinant de présenter et décrire les nombreuses techniques et stratégies mises au point par les membres du groupe de Palo Alto pour entraîner leurs clients à adopter des comportements ridicules ${ }^{3}$. Nous limiterons pourtant ici notre objectif à la recherche des postulats et fondements théoriques sur lesquels repose une telle position.

\section{Postulats et fondements théoriques}

\section{La perception des réalités}

Le premier postulat, et le plus fondamental, du groupe de Palo Alto est que LA vérité, ça n'existe pas, pas plus d'ailleurs que LA réalité :

"Notre conviction la plus fondamentale - de fait, une méta-position, dont toutes nos autres positions découlent - [... est] que tout ce que nous avons ou aurons, ce sont des opinions. Il ne s'agit même pas d'opinions qui soient plus ou moins réelles ou vraies, ou qui se rapprochent progressivement de la vérité. Certaines opinions peuvent être plus utiles ou plus efficaces que d'autres dans l'atteinte d'un but visé." (Fisch et al., 1982: 10-11; traduction de l'auteur.)

Il énonce donc que l'humain organise ses perceptions en une vision du monde selon qu'elle contribue plus ou moins à atteindre ses objectifs. Voilà qui qualifie le statut phénoménologique de sa position scientifique, et dont le groupe de Palo Alto s'attarde à dégager les conséquences utiles et pratiques pour l'intervention d'aide.

Toute personne qui demande de l'aide souffre, d'une façon ou d'une autre, de "sa" relation avec le monde, c'est-à-dire d'une 
contradiction non résolue entre la façon dont elle perçoit les choses et la façon qu'elle voudrait qu'elles lui paraissent, dans le cadre de sa vision du monde.

Cette personne, en difficulté, n'a que deux options: modifier le cours des événements et adapter le monde à son image ou, si ce monde ne peut être modifié, changer la vision qu'elle s'en fait (Watzlawick, 1978 : 40-41).

Une définition plus détaillée est nécessaire, ici, pour saisir toute la complexité et la richesse d'une vision du monde :

Nous devons concevoir une vision du monde [...] comme la synthèse la plus globale et complète qu'un individu peut constituer de ses myriades d'expériences et d'influences, de leur interprétation, et des valeurs et significations attribuées en conséquence aux objets de la perception [...] Ce n'est pas "le monde", mais une mosaïque d'images uniques qu'il est possible d'interpréter d'une façon aujourd'hui et complètement différemment le lendemain; c'est un schème de schèmes; une interprétation des interprétations; le résultat de perpétuelles décisions sur ce que ces méta-interprétations peuvent ou ne peuvent pas embrasser, lesquelles sont elles-mêmes les conséquences de décisions antérieures (Watzlawick, 1978: 43; traduction de l'auteur.)

II n'est donc pas surprenant de constater, avec le groupe de Palo Alto, à quel point il peut être difficile pour un client de vouloir ou pouvoir modifier sa vision du monde, sa réalité, et ce, même s'il peut reconnaître qu'elle constitue un obstacle important à la résolution de son problème.

Avant d'aborder comment l'intervenant procédera, il est nécessaire de comprendre que cette position du groupe de Palo Alto repose sur des fondements théoriques relatifs au fonctionnement du cerveau humain, celui-là même qui est responsable du développement de ces visions du monde. Dans The Language of Change, Watzlawick rapporte les résultats de la recherche neurologique sur le fonctionnement du cerveau et en dégage les conclusions pour l'intervention d'aide. En résumé, la recherche neurologique a permis d'établir que: a) le cerveau humain est constitué de deux hémisphères différents ; $b$ ) chaque hémisphère assume des fonctions différentes; $c$ ) les deux hémisphères fonctionnent simultanément et presque indépendamment l'un de l'autre; d) l'activité interne de chaque hémisphère est plus intense que celle qui relie chaque hémisphère, ce qui rend vraiment très difficile, pour une personne, de traduire le langage de l'un dans le langage propre à l'autre; e) chaque hémisphère interprète à sa façon propre (c'est-à-dire selon sa compétence) un même élément de réalité ; 
et f) celui qui "l'emportera " sur l'autre, pour ainsi dire, sera celui qui, en raison de sa spécialisation, sera plus compétent que l'autre pour traiter plus adéquatement l'élément de réalité en question (Watzlawick, $1978:$ 19-20 et 28-36).

Chez un droitier, les principales fonctions de l'hémisphère gauche (et l'inverse pour le gaucher) ${ }^{4}$ sont de traduire les perceptions en représentations logiques, sémantiques et phonétiques de la réalité, communiquer avec le monde extérieur sur la base d'un codage analytico-logique de l'environnement, et contrôler la psychomotricité. L'hémisphère gauche sert donc à tout ce qui concerne le langage, la pensée "rationnelle", la lecture, l'écriture, le calcul et, en général, la communication digitale.

L'hémisphère droit sert à la compréhension globale de relations complexes, de schèmes, de configurations et de structures; il est responsable de la reconnaissance complète et spontanée d'une réalité, sur la base d'un minimum d'informations. Il assume aussi la fonction d'élaboration de concepts et de types logiques qui ordonnent et clarifient la complexité de la réalité. C'est donc lui qui est responsable de dégager une vision du monde (Watzlawick, 1978: 21-25).

La conclusion pratique qu'il faut tirer de ces résultats de la recherche en neurologie est capitale pour l'intervention d'aide : toute tentative d'influencer le cerveau humain doit s'adresser à l'hémisphère approprié à la circonstance et doit même utiliser le langage de l'hémisphère en question (Watzlawick, 1978: 32).

En termes plus concrets, l'intervention d'aide qui vise à influencer la vision du monde d'un client doit accorder une importance et une attention fondamentales à bien l'identifier et à utiliser cette connaissance pour induire le changement. Pour être efficace, le langage utilisé par l'intervenant ne cherchera pas à traduire le langage analogique (davantage rattaché à l'hémisphère droit, donc à des associations intuitives et à sa vision du monde) du client dans le langage digital (explication, discussion, analyse, confrontation d'opinions et de valeurs, ou interprétation essayant de rendre plus explicite l'inconscient), mais il utilisera des schèmes linguistiques plus appropriés aux capacités de l'hémisphère droit, bloquera l'accès du client à son hémisphère gauche, et prescrira les comportements nécessaires à régler le problème dont se plaint le client (Watzlawick, $1978: 47$ ) et ce, dans des termes compatibles et congruents à sa vision du monde (Fisch et al., 1982: 109). 


\section{L'interaction dans l'ici et maintenant}

Le groupe de Palo Alto est bien connu pour fonder son approche sur la théorie de la communication. Pour les fins de ce travail, ce qu'il faut souligner de la théorie de la communication à laquelle souscrit ce groupe, c'est son postulat interactionnel. L'approche du groupe :

«[...] n'est pas [fondée] sur l'image du télégraphe ou du ping-pong un émetteur envoie un message à un récepteur qui devient à son tour un émetteur, etc., mais sur la métaphore de l'orchestre. La communication est conçue comme un système à plusieurs canaux auquel l'acteur social participe à tout instant, qu'il le veuille ou non : par ses gestes, son regard, son silence, sinon son absence. En sa qualité de membre d'une certaine culture, il fait partie de la communication comme le musicien fait partie de l'orchestre. Mais, dans ce vaste orchestre culturel, il n'y a ni chef, ni partition. Chacun joue en s'accordant sur l'autre." (Winkin, $1981: 7-8$ ).

C'est dans cette perspective interactionnelle qu'il faut comprendre les cinq axiomes de la communication dont le premier, "il est impossible de ne pas communiquer ", marque le ton et souligne très clairement l'orientation, non seulement "comportementale", au sens skinnérien du terme, mais encore et surtout interactionnelle du groupe de Palo Alto (Watzlawick et al., 1967 : 48-71).

Ainsi qu'ils le soulignent explicitement, leur postulat interactionnel exige de centrer l'attention sur la communication interpersonnelle, courante et actuelle, pour expliquer comment les comportements rendent possibles le développement et la persistance des problèmes:

"Sauf pour les cas où des déficiences organiques sont claires et bien déterminées, nous avons proposé que tous les comportements, y compris ceux étiquetés comme "troubles" de comportement, se développent et sont maintenus — "renforcés" au sens général — par d'autres comportements courants ; c'est-à-dire dans l'ici et maintenant, par des rapports humains actuels et significatifs. [... De plus, nous] proposons que le foyer précis de comportements qui maintiennent un problème trouve régulièrement son point central dans les comportements que le patient ou les autres parties concernées ont adoptés dans leurs efforts de contrôler ou régler le problème. C'est dire que nous avons proposé que la persistance - et donc, l'existence même - des problèmes est fondée sur un cercle vicieux de renforcement réciproque entre le comportement problématique d'une part et le comportement adopté comme tentative de "solution" d'autre part. " (Fisch et al., 1982 : 286-287 ; traduction de l'auteur.)

C'est donc en s'appuyant davantage sur les concepts systémiques d'équifinalité et de feedback positif (amplification des écarts) que le 
groupe de Palo Alto explique plus particulièrement l'apparition et le maintien des problèmes: la communication interpersonnelle n'est qu'une longue séquence ininterrompue de comportements ou d'échanges auxquels les participants réagissent, ce qui entraîne en retour la réaction des partenaires. Et si la réaction en chaîne a aussi pour effet d'éloigner les partenaires d'un état initial d'équilibre dynamique, le système risque d'éclater, à moins que de nouvelles réactions de feedback négatif ne permettent de rétablir un nouvel équilibre dynamique (réduction des écarts) (Watzlawick et al., 1967 : 134-148).

Il est notoire que l'approche systémique explique plus facilement la stabilité que le changement, et cela semble encore donner raison à l'adage du "plus ça change, plus c'est pareil ». Mais l'intervention d'aide vise à modifier des comportements et des situations, à induire des changements qui déséquilibrent les systèmes établis, mais pas nécessairement au point de les faire éclater. Pour appuyer encore plus adéquatement sa théorie de la persistance des problèmes et de changement thérapeutique, c'est vers la théorie des ensembles et celle des types logiques, toutes deux provenant de la logique mathématique, que se tourne le groupe de Palo Alto.

\section{Théorie des ensembles et théorie des types logiques}

L'inspiration initiale de puiser dans la théorie des types logiques vient de l'anthropologue Bateson; et le groupe de Palo Alto n'a jamais vraiment abandonné cet héritage (Watzlawick, 1967: 192-228). Mais toute sa pertinence et son utilité deviennent plus évidents lorsqu'elle est juxtaposée à la théorie des ensembles.

En logique mathématique, la théorie des ensembles explore les relations entre les éléments d'un ensemble et l'ensemble lui-même. Elle postule d'un ensemble qu'il a quatre propriétés (Watzlawick et al., 1974: 2-6) :

1. Les éléments d'un ensemble partagent tous au moins une caractéristique en commun et le résultat de toute combinaison des éléments d'un ensemble est lui-même un élément de l'ensemble. Par exemple, chacun des chiffres 1 à 12 d'une horloge est un élément de cet ensemble et toute combinaison de deux éléments produit un résultat qui est lui-même un élément de l'ensemble (ex.: $8 \mathrm{~h}+6 \mathrm{~h}=2 \mathrm{~h}$ de l'après-midi). Cette propriété permet donc de constater qu'une multitude de changements sont possibles à l'intérieur de l'ensemble, et 
impossibles à l'extérieur de l'ensemble, du moins en terme des seuls éléments de celui-ci. Cette activité mentale de regrouper et combiner divers éléments semblables en un tout intégré est une composante indispensable de la perception et des conceptions qu'il est possible de se faire de la réalité. C'est cette activité mentale qui permet d'organiser et de mettre un peu d'ordre dans un univers qui ne serait rien d'autre que pur chaos.

2. Peu importe l'ordre dans lequel les éléments d'un ensemble sont regroupés, le résultat sera le même. Ainsi, à partir d'un point de départ donné, un mouvement d'un kilomètre en direction d'un des quatre points cardinaux suivi du retour au point de départ initial produira le même résultat, quelle que soit la direction dans laquelle les mouvements sont effectués. Cette propriété rend donc compte de la possibilité de changements au niveau des processus, mais ne permet pas de modifier l'état final.

3. Un ensemble comprend un élément neutre (identity number), de telle sorte que, combiné à tout autre élément de l'ensemble, le résultat est égal à ce dernier. Ainsi, dans un ensemble dont la règle de combinaison est l'addition, l'élément neutre est zéro (par exemple : $5+0=5$ ); dans un ensemble dont la règle est la multiplication, l'élément neutre est $1(5 \times 1=5)$. Enfin, si l'on considère les sons comme un ensemble, l'élément neutre serait le silence. C'est ainsi que l'élément neutre permet aux autres éléments de l'ensemble de maintenir leur identité respective. Cette propriété permet aussi de comprendre comment un membre d'un groupe peut agir sans affecter les autres membres, comme dans les situations où un membre nie l'existence d'un problème.

4. Tout élément d'un ensemble a sa réciproque, de telle sorte que le résultat d'une combinaison d'un élément et de sa réciproque produit un résultat égal à son élément neutre : ainsi $5+(-5)=0$, dans un ensemble dont la règle de combinaison est l'addition. $\mathrm{Si}$ cette propriété illustre qu'un changement radical peut survenir dans l'ensemble, le résultat est encore un élément de l'ensemble. Tandis que la propriété de l'élément neutre est celle qui permet à un élément de maintenir son identité propre, la propriété de la réciproque est celle qui permet à l'ensemble de maintenir son identité propre, en dépit de modifications qui affectent les éléments dans leurs interrelations.

L'intérêt de cette théorie pour le groupe de Palo Alto, est qu'elle approfondit et précise les concepts systémiques de morphogénèse et 
de morphostase, en identifiant clairement comment des changements de toutes sortes peuvent survenir à l'intérieur d'un ensemble sans pour autant modifier l'état final de l'ensemble : "plus ça change, plus c'est pareil».

Pour expliquer quels types de changements et comment des changements peuvent modifier l'ensemble comme ensemble, c'est vers la théorie des types logiques qu'il faut se tourner (Watzlawick et al., $1974: 6-7)$. À son tour, cette théorie postule, au départ, une collection d'éléments qui partagent un dénominateur commun. Ces collections portent ici le nom de "classes", mais pourraient tout aussi bien s'appeler "systèmes" ou "ensembles». L'axiome central de cette théorie est fort simple : une classe ne peut être membre de sa classe ; aussi, tout effort de traiter l'une en termes propres à l'autre ne saurait qu'aboutir à de la confusion. Ainsi l'humanité, comme classe des êtres humains, ne saurait elle-même être un être humain. Cette propriété s'apparente aussi à la propriété holistique des systèmes selon laquelle le tout dépasse la somme des parties.

II reste à voir comment "les types logiques" sont reliés au changement. Une des formes les plus élémentaires de changement, c'est un mouvement, du moins en ce qu'il suppose la modification d'une position. Mais le mouvement peut lui-même faire l'objet d'un changement, et l'accélération en est une bonne illustration. Aussi, pour passer du concept de " position » à celui de " mouvement ", il faut passer à un niveau d'abstraction hiérarchiquement supérieur au premier. Il en va de même pour passer du concept de "mouvement" à celui "d'accélération ». Pour traiter et parler d'une classe, il faut donc sortir et quitter cette classe ; il faut "métacommuniquer " à propos d'elle.

Accepter la différence et la complémentarité de ces deux théories, c'est reconnaître qu'il existe deux types de changements : un premier qui survient à l'intérieur d'un système qui reste lui-même inchangé ; et un deuxième dont l'introduction modifie le système lui-même. Par exemple, la personne qui a un cauchemar peut s'adonner à diverses activités dans son rêve, mais le passage d'une activité à l'autre, dans ce rêve, ne pourrait à elle seule mettre fin au cauchemar lui-même. C'est à ce type de changement (le passage d'une activité à l'autre sans mettre fin au rêve) que le groupe de Palo Alto pense lorsqu'il est question de "changement de premier niveau"; la seule façon de "sortir" du cauchemar nécessite de passer de l'état de rêve à celui d'éveil; se réveiller ne fait pas partie du rêve et le transcende, c'est ce type de changement que le groupe de Palo Alto nommera "changement de deuxième niveau " (Watzlawick et al., 1974 : 10). 
"Plus ça change, plus c'est pareil » sera donc du premier degré, et les systèmes ne subiront aucun véritable changement lorsqu'il sera question d'un tel ordre de changement. Pour qu'un véritable changement puisse s'opérer, du moins en ce qui concerne la résolution des problèmes, c'est donc au deuxième niveau qu'il faudra en appeler, un type de changement qui nécessitera une discontinuité, un saut d'un niveau d'abstraction à un autre, et qui ne pourra donc éviter de surprendre ou paraître différent, et ce à un point tel qu'il paraîtra même illogique et paradoxal (Watzlawick et al., 1974 : 11).

Les difficultés évolueront donc en problèmes par l'application de solutions qui relèveront de changements du premier ordre, selon l'une ou l'autre des trois façons suivantes (Watzlawick et al., 1974 : 38-39) :

a) la mesure appliquée est de nier la réalité d'un problème : elle est nécessaire mais n'est pas entreprise ;

b) une mesure est prise qui tente de régler un problème impossible à résoudre: une mesure est prise quand elle ne devrait pas l'être ; et

c) une mesure est prise, mais au mauvais niveau d'abstraction, soit qu'elle saute à un palier supérieur quand elle devrait rester au même palier (par exemple: demander un changement d'attitude quand un changement de comportement suffirait), ou qu'elle est tentée au même niveau quand elle devrait se situer à un autre (comme dans le cas du cauchemar ou de l'insomniaque).

L'insistance à tenter de modifier ou régler une situation pénible par une solution inadéquate est précisément ce qui renforce la difficulté et l'intensifie au point qu'elle peut évoluer en un problème insupportable.

La résolution du problème requiert donc la mise en place de comportements qui, différents et nouveaux, relèveront d'un changement de deuxième ordre, et rompront les cercles vicieux établis, par leur caractère de discontinuité, d'illogisme, de paradoxe ou d'absence de sens commun. Ce qu'il faut souligner, c'est que le règlement du problème ne s'obtiendra pas en s'attaquant au problème comme tel ou à ses causes profondes, mais bien plutôt en s'attaquant aux essais infructueux du client (Watzlawick, $1974: 81$ ).

En résumé, quatre principes se dégagent du point de vue du changement de deuxième ordre (Watzlawick, 1974: 82-3) :

a) il vise ce qui, dans la perspective du changement de premier ordre, semblait une solution, parce que, dans sa perspective, c'est cette solution qui est le nœud du problème (le problème n'est vraiment rien d'autre que sa solution); 
b) si un changement de premier ordre paraît fondé sur le sens commun, la discontinuité du changement de deuxième ordre confère à celui-ci un caractère étrange et illogique ;

c) la mise en place d'un changement de deuxième ordre vise à modifier des comportements actuels et non ses causes historiques, lointaines, ou "profondes".

d) I'utilisation de techniques qui provoquent un changement de deuxième ordre débloque du piège la situation pénible qu'engendraient les cercles vicieux des changements de premier ordre, et la situe à un nouveau niveau d'abstraction, ou dans une nouvelle vision du monde, plus utile et bénéfique.

Sans vouloir élaborer sur les techniques et stratégies qui peuvent susciter un changement de deuxième ordre, il faut rappeler l'importance fondamentale de bien connaître la vision du monde du client et s'en servir. Cela permettra d'obtenir plus facilement son adhésion aux prescriptions comportementales de l'intervenant, qui se feront surtout par le truchement de la "reformulation dirigée", qui, elle, consiste à soumettre au client une vision du monde différente de celle qu'il avait ; mais il y a aussi des éléments de continuité, de congruence et de compatibilité; on tentera de dégager l'intention positive ou plus acceptable que le client et ses partenaires poursuivent, ou de préciser la menace dont ils cherchent à se protéger selon, par exemple, que la vision qu'ils ont du problème fait voir ce dernier en termes ou de maladie ou de mauvaise foi (Watzlawick et al., 1974: 95-99).

\section{Conclusion}

L'analyse théorique du changement en relation d'aide présentée dans cet article peut paraître rébarbative et sans intérêt immédiat pour la pratique elle-même. Et pourtant, ceux qui sont familiers avec les stratégies et les techniques de "thérapie brève" auront reconnu la grande pertinence de ces éléments théoriques qui appuient les interventions "brèves " et facilitent aussi la recherche plus méthodique et éclairée de stratégies et techniques plus raffinées et efficaces.

Le postulat interactionnel du groupe de Palo Alto fonde aussi l'intervention "sociale" plus clairement que les postulats plus psychologiques ou psychiatriques de nombreuses autres approches. Sans pour autant renier certains éléments de ces autres approches, il rappelle très concrètement que les gens éprouvent des problèmes dont l'origine se trouve dans leurs rapports actuels avec d'autres personnes, 
et pas seulement ou nécessairement dans des carences ou déficiences personnelles. L'associer à celui sur la perception des réalités facilite, encourage et exige même très concrètement une intervention d'aide profondément respectueuse des valeurs, opinions et sentiments du client: il ne s'agit plus d'un vœu qui peut rester pieux mais d'une démarche qui repose sur, et qui doit recourir à la vision du monde du client plutôt qu'à celle de l'intervenant.

À sa position interactionnelle, le groupe de Palo Alto combine aussi une position systémique, et met donc l'accent sur les processus circulaires et sur l'équifinalité de la communication interpersonnelle. Une telle approche permet d'introduire des comportements qui rompent des cercles vicieux et, en même temps, amorcent de nouveaux cercles, ceux-ci plus "bénéfiques". Ces nouveaux comportements n'ont pas besoin d'être dramatiques ou majeurs; au contraire, il leur suffit, ou mieux encore, il importe presque qu'ils frisent la banalité pourvu que leur introduction dans le système soit stratégique et bien planifiée. Une fois amorcés ces nouveaux cercles bénéfiques, l'intervenant peut se retirer et miser sur les suites des comportements initiés pour qu'ils fassent "boule de neige». Sur le plan éthique, une telle position permet aussi de croire en des interventions plus efficaces pour atteindre des résultats, et plus efficientes parce que plus brèves et donc moins coûteuses en temps et en énergie.

Au terme de cet exposé, il ne faut pas croire, qu'à elle seule, l'approche de thérapie brève du groupe de Palo Alto permet la résolution de tous les problèmes soumis par les clients. En effet, les textes produits par ce groupe laissent croire que tout problème est le résultat d'interactions entre diverses personnes. Une telle position, parce que trop catégorique, peut devenir dangereuse: différents milieux ou contextes de vie échappent au contrôle et à la responsabilité des individus et peuvent influencer leurs comportements à un point où ces individus vivent des problèmes très réels et pénibles qui les dépassent. Qu'il suffise de penser au phénomène du chômage, de la pauvreté, à la guerre, à la pollution... Il s'agit là de problèmes qui ont des incidences directes sur le bien-être d'innombrables personnes et que l'intervenant social ne saurait résoudre avec la seule approche du groupe de Palo Alto.

\section{Notes et références}

* L'auteur tient à souligner l'apport important de Hugues Grenier et Pierre Roberge, professeurs en travail social et de Jacques Charest, professeur en 
psychologie à l'Université du Québec en Abitibi-Témiscamingue dont les commentaires et les réflexions ont grandement contribué à enrichir la rédaction de cet article.

1 Voir à ce sujet l'excellent ouvrage de Yves WINKIN, La nouvelle communication, Paris, Seuil, 1981, pp. 27-61.

2 Cette section constitue un résumé fidèle de FisCH et al., 1982 : 12-14, 287-288 et s'inspire aussi de WEAKLAND et al., 1974 : 147-150 et de WATZLAWICK, 1974 : 1-2, 34-39.

3 Ces techniques et stratégies sont rapportées de façon détaillée dans FisCH et al., 1982: 21-188 et Herr et WeakLAND, 1979: 12-173, comme des étapes d'intervention.

4 Si cette relation de l'inversion latérale des hémisphères prévaut encore, quelques travaux neurologiques rapportés par Watzlawick permettent maintenant de questionner cette hypothèse (WATZLAWICK, 1978, 20).

\section{Références bibliographiques}

Fisch, R., J.H. WeakLand et L. SeGal, The Tactics of Change: Doing Therapy Briefly, San Francisco, Jossey-Bass, 1982, 302p.

GarneaU, J. et M. LARIVEY, L'auto-développement: psychothérapie dans la vie quotidienne, Outremont, Ressources en développement, 1979, 332p.

Herr, J.J. et J.H. Weakland, Counseling Elders and Their Families, New York, Springer, 1979, 308p.

SeGAL, L., "Brief family therapy", dans: A.M. Horne et M.M. OhtSen, Family Counseling and Therapy, Itasca, (III.), Peacock, 1982.

Watzlawick, P., H.J. Beavin et D.D. Jackson, Pragmatics of Human Communication : A Study of Interactional Patterns, Pathologies and Paradoxes, New York, Norton, 1967, 294p.

Watzlawick, P., J. Weakland et R. Fisch, Change: Principles of Problem Formation and Problem Resolution, New York, Norton, 1974, 172p.

WATZLAWICK, P., The Language of Change : Elements of Therapeutic Communication, New York, Basic Books, 1978, 172p.

WeAkland, J.H., R. FisCh, P. WATZlaWick et M.A. Bodin, "Brief therapy: Focused problem resolution", Family Process, vol. 13, no 2, 1974 : 141-168.

WinkIN, Y. (éd.), La nouvelle communication, Paris, Seuil, 1981, 382p. ("Points", 136.) 